\title{
Loss of dipeptidyl peptidase IV immunostaining discriminates malignant melanomas from deep penetrating nevi
}

\author{
Alexander Roesch, Sina Wittschier, Bernd Becker, Michael Landthaler and Thomas Vogt \\ Department of Dermatology, University of Regensburg, Regensburg, Germany
}

\begin{abstract}
The deep penetrating nevus is a rare variant of benign melanocytic nevus with histologic features mimicking vertical growth phase, nodular malignant melanoma. In this study, we expand on the search for new complementary discriminating markers by analyzing a selection of both cell cycle-related factors, such as retinoblastoma protein and phospho-retinoblastoma protein Ser795 as indicators for retinoblastoma protein activation/inactivation status, and invasion-related factors, such as matrix metalloproteinase-1, matrix metalloproteinase-2, membrane-type matrix metalloproteinase-1 and integrin $\beta 3$. MIB-1/Ki-67 was analyzed as an example for a common proliferation marker. Dipeptidyl peptidase IV/CD26 was analyzed as a marker affecting both proliferation and invasion of malignant melanocytic tumors. Semiquantitative assessment of both immunolocalization and immunoreactivity of retinoblastoma protein and phospho-retinoblastoma protein Ser795, MIB-1/Ki-67, matrix metalloproteinase-1, matrix metalloproteinase-2, membrane-type matrix metalloproteinase-1 and integrin $\beta 3$ revealed no consistent differences between deep penetrating nevi $(n=14)$ and matched cases of nodular malignant melanomas $(n=10)$. Matrix metalloproteinase-1 and matrix metalloproteinase-2 immunostaining of some deep penetrating nevi even exceeded that of nodular malignant melanomas. Membrane-type matrix metalloproteinase-1 expression scores of nodular malignant melanomas were higher than those of deep penetrating nevi, which was, however, not significantly discriminative. In contrast, immunostaining of dipeptidyl peptidase IV was significantly discriminative due to a consistent lack of dipeptidyl peptidase IV-expression in nodular malignant melanomas. These results add evidence that among the selected markers supposed to be relevant for melanoma progression the presence of dipeptidyl peptidase IV can be used to support diagnosis of deep penetrating nevi in doubtful cases. As loss of dipeptidyl peptidase IV may also be causally linked to the transition of invasive to metastatic phenotypes, the molecular mechanisms downstream of dipeptidyl peptidase IV deserve to be studied in more detail in future investigations.
\end{abstract} Modern Pathology (2006) 19, 1378-1385. doi:10.1038/modpathol.3800663; published online 7 July 2006

Keywords: melanoma; deep penetrating nevus; dipeptidyl peptidase IV; CD26; matrix metalloproteinases; cell cycle

In 1989 , Seab et $a l^{1}$ reported a series of benign invasive pigmented melanocytic tumors and coined the term deep penetrating nevus. Barnhill et $a l^{2,3}$ reported a histomorphologically similar melanocytic lesion using the term plexiform spindle cell nevus. However, the plexiform spindle cell nevus is more plaque-like and surrounds the superficial neurovascular plexus. Deep penetrating nevi mostly appear as darkly pigmented papules or nodules with no or mild epidermal changes. They are most commonly found in the face, on the upper trunk or proximal extremities of patients at the age of 10-30

Correspondence: Dr A Roesch, MD, Department of Dermatology, University of Regensburg, Franz-Josef Strauss-Allee 11, D-93053 Regensburg, Germany.

E-mail: alexander.roesch@klinik.uni-regensburg.de

Received 26 April 2006; revised and accepted 15 June 2006; published online 7 July 2006 years. ${ }^{1,4}$ Histopathologic growth patterns are often worrisome showing a wedge-shaped invasive growth extending from the upper dermis into the subcutaneous fat tissue not rarely following preformed structures, for example hair follicles or sweat glands. ${ }^{1,5}$

Cytological pleomorphism defined as a variation of cell size and shape together with hyperchromasia, one hallmark of malignant melanomas, is reported to occur also in the deeper, invasive portions of deep penetrating nevi. ${ }^{5}$ Further deep penetrating nevus features that complicate the distinction from malignant melanoma are the presence of inflammatory stroma reactions observed in $75 \%$ of deep penetrating nevi, lack of melanocytic maturation in the deep portion and even some possible degree of lesional asymmetry. $^{4-6}$ In some cases, only histological features together with clinical follow-up information can confirm that a lesion was truly benign. 
Thus, as several studies have emphasized, deep penetrating nevi are often mistaken for vertical growth phase nodular malignant melanomas, both clinically and histologically. ${ }^{1,2,5-70}$ Estimates exist that, depending on the criteria used for classification, misdiagnoses as melanoma occur in $29-40 \%$ of the cases. ${ }^{1,4,5}$ The opposite, that is taking advanced melanomas as deep penetrating nevi may also occur.

Unfortunately, to this end immunohistochemical analyses also failed to differentiate deep penetrating nevus from nodular malignant melanoma. ${ }^{1,11}$ To the best of our knowledge, to date, only one study exists suggesting PCNA (proliferating cell nuclear antigen) as a possibly discriminating marker. ${ }^{12}$ PCNA represents an accessory protein of DNA $\delta$-polymerase which is increased during the late G1 growth phase and peaks in the $S$ phase of the cellular cycle.

Beyond the clinical demand for precise diagnosis, the deep penetrating nevus may also represent a valuable natural model for melanocytic invasion without metastatic potential, thereby, querying the role of MMPs in melanomas. Here, we expand on the search for complementary discriminating markers analyzing either $\mathrm{pRb} /$ phospho-pRb and MIB-1/Ki-67 as a direct read-out of cell cycle progression and proliferation or invasion-related proteins such as MMPs or, finally, a marker related to invasion as well as proliferation, DPPIV.

\section{Materials and methods}

\section{Tissue Samples and Immunohistochemistry}

Tumor material and clinical follow-up information of 14 deep penetrating nevi and a set of matched nodular malignant melanomas $(n=12)$ was collected at the Department of Dermatology, University of Regensburg, Germany. Written consent of the patients was obtained prior to surgery. Conventional paraffin embedded tissue sections were deparaffinized and rehydrated according to standard protocols. After inhibition of endogenous peroxidase activity with hydrogen peroxide, three different protocols for antigen retrieval were performed: (1) For MIB-1/Ki-67, integrin $\beta 3-$, phospho-pRb Ser795and total $\mathrm{pRb}$-staining, the sections were incubated at $100^{\circ} \mathrm{C}$ for $30 \mathrm{~min}$ with citrate buffer ( $\mathrm{pH} \mathrm{6.0)}$. (2) For DPPIV-detection, EDTA buffer (pH 8.0) was used. (3) MMP-1-, MMP-2 and MT1-MMP-staining required pretreatment with $150 \mu \mathrm{l}$ pepsin for $25 \mathrm{~min}$ at $37^{\circ} \mathrm{C}$. After washing with PBS, samples were blocked with SuperBlock ${ }^{\mathrm{TM}}$ (Zytomed, Berlin, Germany) and subsequently incubated with a 1:100 dilution of the primary antibody for $35 \mathrm{~min}$ at $37^{\circ} \mathrm{C}$. For detection of total $\mathrm{pRb}$ and phosphorylated $\mathrm{pRb}$, $R b$ (4H1) Monoclonal antibody and Phospho-Rb (Ser795) Monoclonal antibody, respectively, were used from Cell Signaling Technology/New England Biolabs GmbH, Frankfurt, Germany. Ki-67 was detected by the MIB-1 antibody, DakoCytomation, Glostrup, Denmark. Integrin $\beta 3-$, MMP-1-, MMP-2- and MT1-MMP-detection was performed with anti-human-CD61 (CBL479)-, anti-human-MMP-1 (MAB3307)-, anti-human-MMP-2 (MAB13405)- and anti-human-MT1-MMP (MAB3317)-antibodies from Chemicon, Hampshire, UK. Anti-human-CD26 (D068-1) antibody from MBL, Woburn, USA was used for DPPIV-staining. For detection of all primary antibodies, the avidin-biotin complex method was performed using a biotinylated secondary antibody (ZytoChemPlus Anti-Broad Spectrum ${ }^{\mathrm{TM}}$ ) together with avidin-conjugated horseradish peroxidase (ZytoChemPlus HRP ${ }^{\mathrm{TM}}$ Zytomed, Berlin, Germany) and AEC Substrate Chromogen ${ }^{\mathrm{TM}}$ (DakoCytomation, Hamburg, Germany) according to the manufacturers' recommendations.

\section{Immunohistochemical Evaluation}

To reduce bias, immunostaining was assessed by two independent investigators (AR, SW) in a blinded fashion. Inter- and intra-examiner reproducibility was 82 and $88 \%$, respectively. Immunoreactivity was scored using uniform criteria to maintain the reproducibility of the method. The relative quantity of immunostaining was recorded considering the expression in tumor cells (nuclear, cytoplasmatic or membranous staining) as well as in the surrounding extracellular matrix (in case of MMPs).

Considering nuclear staining of total $\mathrm{pRb}, \mathrm{pRb}$ Ser795 and MIB-1/Ki-67, for each sample, three representative fields of vision were evaluated at $\times 400$ magnification in a tumor region with maximum and a region with minimum staining as described previously. ${ }^{13,14}$ Briefly, the number of positive stained nuclei was estimated as percentage of all nuclei $(p)$ per field of vision. In addition, the staining intensities (i) of positive cells were scaled from grade 1, when the cells showed a slight granular staining pattern, to grade 4 , when the cells had completely filled nuclei. Grades 2 and 3 were assigned to intermediate staining intensities. Subsequently, a maximum and minimum expression score $\left(\mathrm{ES}_{\max }, \mathrm{ES}_{\min }\right)$ were calculated for each tumor sample according to following formula shown below. To facilitate comparability among different markers, in this study, the expression information of the whole tumor $\left(\mathrm{ES}_{\text {total }}\right)$ was calculated as average value from $\mathrm{ES}_{\max }$ and $\mathrm{ES}_{\text {min }}$.

$$
\begin{gathered}
\mathrm{ES}_{\text {max }}=\frac{\left(p_{1}+p_{2}+p_{3}\right)}{3} \times \frac{\left(i_{1}+i_{2}+i_{3}\right)}{3} \\
\mathrm{ES}_{\text {min }}=\frac{\left(p_{1}+p_{2}+p_{3}\right)}{3} \times \frac{\left(i_{1}+i_{2}+i_{3}\right)}{3} \\
\mathrm{ES}_{\text {total }}=\frac{\mathrm{ES}_{\text {max }}+\mathrm{ES}_{\text {min }}}{2}
\end{gathered}
$$

$p_{\mathrm{x}}$ is the percentage of stained cells per field of vision $\mathrm{X}, i_{\mathrm{x}}$ the staining intensity of positive 
cells per fields of vision X, ES the expression score.

Integrin $\beta 3$ - and DPPIV exhibit a more complex staining pattern in both entities the deep penetrating nevus and nodular malignant melanoma, respectively. Consequently, the mean staining percentage $(p)$ and intensity (i) were semiquantitatively assessed considering the whole tumor section and not selected fields of vision. Total expression scores $\left(\mathrm{ES}_{\text {total }}\right)$ were calculated in accordance with the algorithm shown above.

Concerning MMP-1, MMP-2 und MT1-MMP also the whole tumor section including stromal surroundings was considered. However, the staining intensity (i) was not taken into account because intensities were invariable if staining was possible.

$$
\mathrm{ES}_{\text {total }}=\frac{\left(p_{1}+p_{2}+p_{3}\right)}{3}
$$

\section{Statistical Analysis}

The SPSS 13.0 (SPSS Inc, Erkrath, Germany) software package was used to perform statistical analyses. For assessment of relative protein expression levels the nonparametric Mann-Whitney $U$-test was applied.

\section{Results}

\section{Immunohistochemical Staining of Cellular Proliferation Markers: Consistency with Previous Studies, but Inapplicability as Discriminating Markers}

Since a previous study has suggested differential expression of $\mathrm{PCNA}^{12}$ in deep penetrating nevi vs melanomas, we were interested if this is also true for the common proliferation marker MIB-1/Ki-67 which is expressed from the mid-G1 phase of the cell cycle through S, G2 and M-phases and the major central cell cycle regulator, $\mathrm{pRb}$. As reported recently by our group, a paradox progressive overexpression of the cell cycle controlling $\mathrm{pRb}$ and its increased inactivation due to phosphorylation are hallmarks of malignant melanomas. ${ }^{13}$

According to our former observations, ${ }^{13}$ there was an intratumoral heterogeneity of total $\mathrm{pRb}$ and phospho-pRb Ser795 expression in nodular malignant melanomas. Particularly at the subepidermal lateral and dermo-invasive portions, both markers revealed high expression scores in most samples $\left(E S_{\max }>100\right)$. In contrast to our previous findings on common benign nevi, ${ }^{13} 33 \%$ of the deep penetrating nevus specimens showed a melanoma-like high deep dermal total $\mathrm{pRb}$ expression with $\mathrm{ES}_{\max }>100$. Furthermore, even $50 \%$ of the deep penetrating nevi evinced a dermal infiltration of phospho-pRb Ser795 positive cells indicating inactive $\mathrm{pRb}$, that is a cell cycle permissive status, very similar to nodular malignant melanomas. In addition to this accumulation of immunoreactivity in the tumor periphery, the majority of deep penetrating nevi showed staining signals for both markers in a more diffusely distributed pattern. In case of $\mathrm{pRb}, 67 \%$ and, in case of phospho-pRb Ser795, 80\% of the deep penetrating nevi evinced such diffuse immunolocalization.

The determination of total expression scores $\left(\mathrm{ES}_{\text {total }}\right.$, see Materials and methods) revealed a higher expression of $\mathrm{pRb}$ and phospho-pRb Ser795 in nodular malignant melanomas (mean $E S_{\text {total-pRb }} 143$, mean $E S_{\text {total }-\mathrm{p}-\mathrm{pRb}}$ Ser795 63 ) than in deep penetrating nevi (mean $\mathrm{ES}_{\text {total-pRb }} 70$, mean $\mathrm{ES}_{\text {total-p-pRb Ser795 }} 53$ ), a finding consistent with our latest data on common benign nevi vs nodular malignant melanomas ${ }^{13}$ and with previous data on PCNA $^{12}$ (Figure 1a and b). Since this differential regulation was only significant for total $\mathrm{pRb}$ $\left(P_{\mathrm{pRb}}<0.005, P_{\mathrm{p}-\mathrm{pRb} \text { Ser795 }}<0.1\right)$, applicability of the $\mathrm{pRb} /$ phospho-pRb ratio as discriminative marker for deep penetrating nevi remains doubtful.

Also the evaluation of MIB-1/Ki-67 staining revealed a statistically significant trend to higher

\footnotetext{
Figure 1 Comparative immunohistochemical staining of cell proliferation-related factors, such as retinoblastoma protein (pRb), phospho-pRb Ser795 and MIB-1/Ki-67, as well as invasion-related factors, such as matrix metalloproteinase-1 (MMP-1), MMP-2, MT1MMP and integrin $\beta 3$. Dipeptidyl peptidase IV/CD26 (DPPIV) was analyzed as a marker affecting both proliferation and invasion of malignant melanocytic tumors. (a) Semiquantitative evaluation of total pRb staining of 12 deep penetrating nevi and 9 nodular malignant melanomas shown as percentage of samples within three categories of expression scores (for definition see Materials and methods). There is a statistically significant trend to higher scores in nodular malignant melanomas $(U$-test $P<0.005)$, but practically this marker might not be applicable for differentiation due to its broad intra- and intertumoral heterogeneity in immunoreactivity. (b) Evaluation of phospho pRb Ser795 staining within four categories of expression scores. There is a trend to higher scores in nodular malignant melanomas, but also not applicable for differentiation ( $U$-test: $P<0.1$ ). (c) Evaluation of MIB-1/Ki-67 staining shows a statistically significant trend to higher scores in nodular malignant melanomas $(U$-test $P<0.001)$. However, practically this marker might not be applicable for differentiation due to its high intertumoral expression variance. (d) MMP-1 expression scores showing a statistically significant trend to increased expression in deep penetrating nevi compared to nodular malignant melanomas $(U$-test $P<0.04)$. (e) MMP2 expression scores suggesting an increased expression in deep penetrating nevi, however, also not applicable due to lacking statistical significance $(U$-test $P<0.6$ ). (f) Increase of the overall MT1-MMP expression in nodular malignant melanomas compared to deep penetrating nevi, statistically not significant ( $U$-test $P<0.1$ ), probably due to broad inter- and intratumoral variance. (g) 'Melanomamimicking' integrin $\beta 3$ expression in deep penetrating nevi, that is not significant based on the ES values $(U$-test $P<0.3)$. (h) DPPIV truly discriminates between deep penetrating nevi and nodular malignant melanomas $(U$-test $P<0.001)$. In contrast to all other markers analyzed, only DPPIV shows both a consistent immunoreactivity, that is low intertumoral heterogeneity, as well as a high statistical significance.
} 
scores in melanomas (mean $\mathrm{ES}_{\text {total-MIB-1/Ki-67 }}$ was 31 for nodular malignant melanomas $v s$ three for deep penetrating nevi, $U$-test $P<0.001)$. However, practically this marker might not be applicable for differentiation due to its high intertumoral expression variance in melanomas (SD for nodular malignant melanomas was 51).

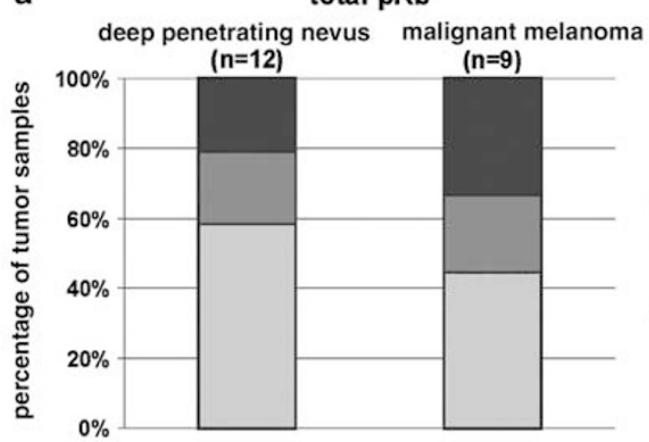

C

MIB-1/Ki-67

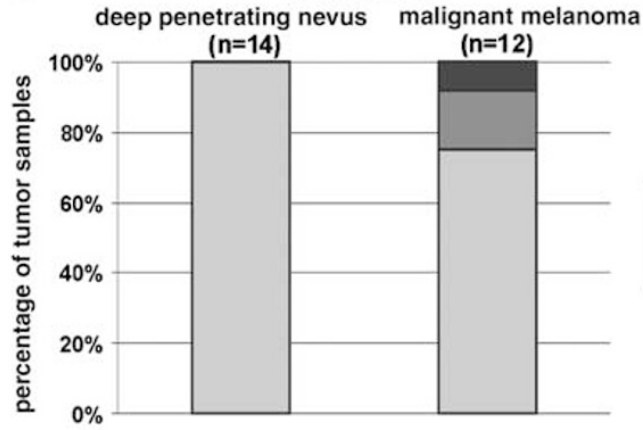

MMP-2

e

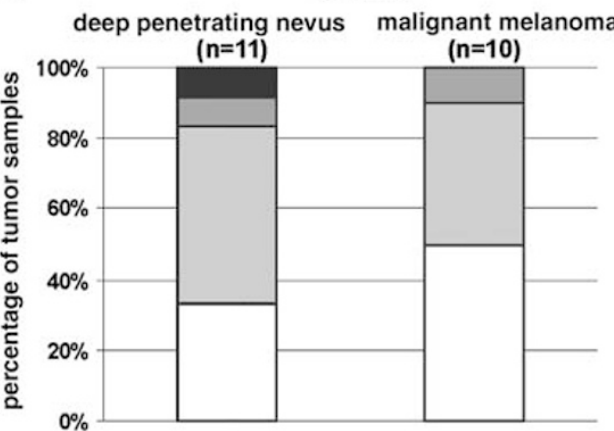

모 $>100$

口ES $50-100$

QES 0.50

$p<0.001$

$\square \mathrm{ES}>50$

IES $20-50$

DES $0-20$

DES 0

$p<0.6$

g

integrin $\beta 3$
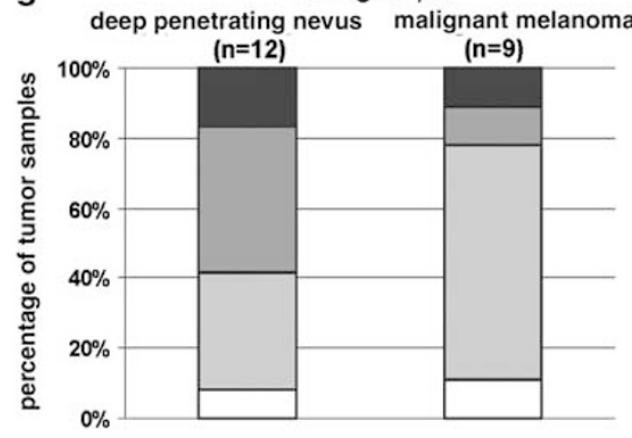

Immunohistochemical Staining of Markers

Representing Invasive Potential: New Aspects of Differential Expression, but Unclear Applicability as Discriminating Markers

Degradation and remodeling of the tumor surrounding extracellular matrix is considered as an essential step in melanoma progression. As repeatedly described,
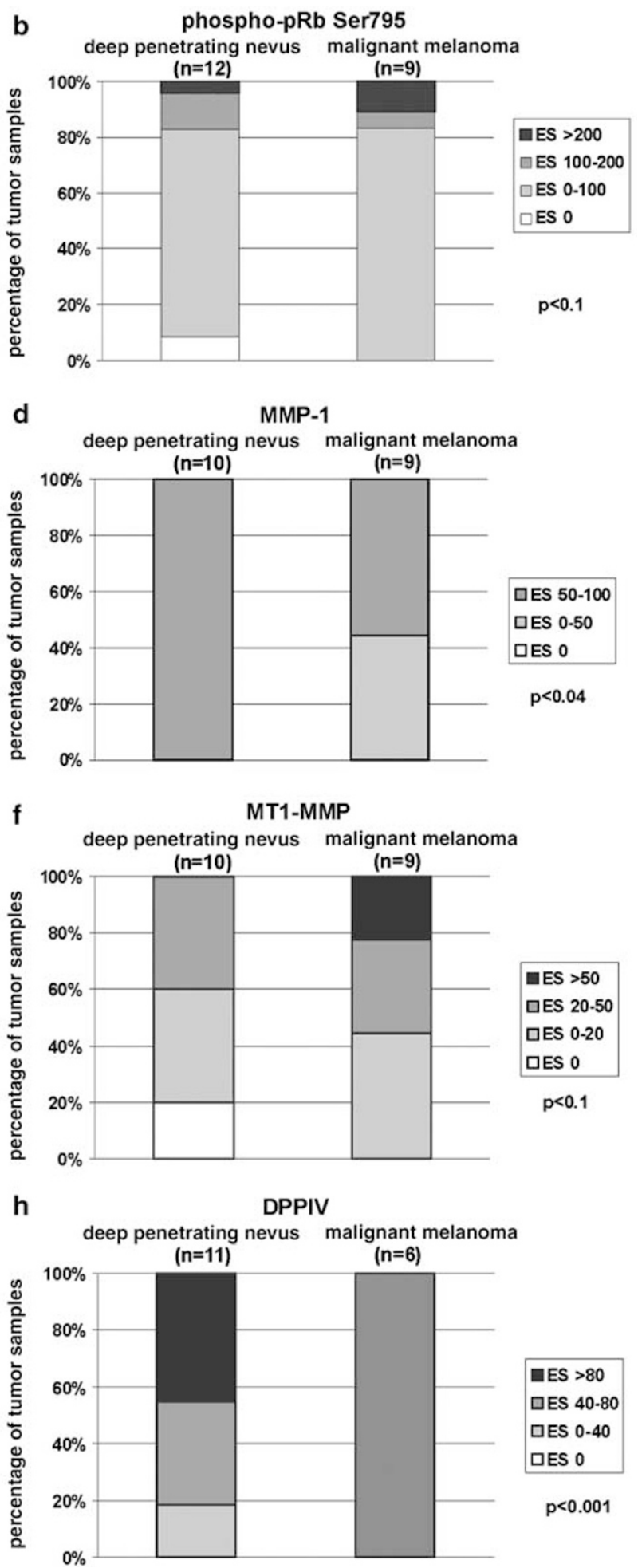
the increased coexpression of a subset of matrix metalloproteinases (MMP-2, MT1-MMP) and integrin $\beta 3$ is correlated with early melanoma invasion. ${ }^{15-18}$ The inductio $\mathrm{n}$ of MMP-1 is reported to be a late event marker during cell invasion of advanced melanomas. ${ }^{19}$

The signal distribution of MMP-1, MMP-2, MT1MMP and integrin $\beta 3$ revealed a complex expression pattern, notably in both analyzed entities. Beside high expression signals at the dermo-invasive front, most tumor samples additionally showed a focal staining pattern in association with cutaneous adnexes and sometimes a more diffuse staining pattern was observed. Concerning the discriminative power, none of the staining patterns, that is focal, diffuse or dermo-invasive, could validly separate deep penetrating nevi from nodular malignant melanomas. Interestingly, the invasive front staining of MMP-2 and integrin $\beta 3$, seen in 46 and $36 \%$ of the deep penetrating nevi, even exceeded the fraction of positive nodular malignant melanomas (20 and 11\%). Subepidermal MT1-MMP signals were found in $78 \%$ of all nodular malignant melanomas vs $10 \%$ of deep penetrating nevi.

Concerning the relative distribution of total expression scores, integrin $\beta 3$ (Figure 1f) and MMP-1 (Figure 1c) appeared approximately equal within both entities. According to this, the mean $\mathrm{ES}_{\text {total-integrin } \beta 3}$ of both entities did not differ significantly $(P<0.3)$. The comparative analysis of $\mathrm{ES}_{\text {total-MMP-1 }}$ of deep penetrating nevi and nodular malignant melanomas only revealed a weak upregulation in deep penetrating nevi with $P<0.04$. Semiquantitative expression of MMP-2 in deep penetrating nevi (mean $\mathrm{ES}_{\text {total-MMP-2 }} 14$ ) even exceeded that of nodular malignant melanomas twice as much (mean $\mathrm{ES}_{\text {total-MMP-2 }}$ 8, Figure 1d). However, since this difference could not reach statistical significance $(P<0.6)$, this parameter seems also not applicable for discrimination. The relative distribution of MT1-MMP expression scores showed an inverse staining result with higher values in melanoma samples (mean $\mathrm{ES}_{\text {total-MT1-MMP }} 36$ ) than in

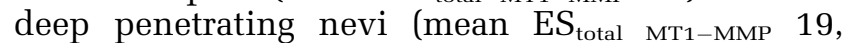
Figure 1e), but this regulation also failed to reach statistical significance $(P<0.1)$.

\section{Immunohistochemical Staining of DPPIV: a Possible Marker for Discrimination Between Deep Penetrating Nevi and Nodular Malignant Melanomas}

Previous studies have shown that DPPIV is almost invariably lost during melanoma progression, which may affect both melanoma proliferation and invasion. ${ }^{20-22}$

In contrast to the other markers in this study, DPPIV staining clearly discriminates the two entities (Figure 1g). The mean $\mathrm{ES}_{\text {total-DPPIV }}$ for deep penetrating nevi (87) significantly exceeded the mean $\mathrm{ES}_{\text {total-DPPIv }}$ of nodular malignant melanomas (9) with $P<0.001$. All deep penetrating nevi stained positive. $46 \%(n=5)$ of all deep penetrating nevi showed an $\mathrm{ES}_{\text {total-DPPIV }}>80$ and $36 \%(n=4)$ revealed an $\mathrm{ES}_{\text {total }}$ between 80 and $40.18 \% \quad(n=2)$ had an $\mathrm{ES}_{\text {total }}$ lower than 40. In contrast, the highest $\mathrm{ES}_{\text {total }}$ reached in a nodular malignant melanoma was 38. All other melanoma samples showed expression scores lower than 10. The intratumoral immunolocalization of DPPIV in both entities showed a diffuse expression pattern with focal accumulation close to cutaneous adnexes or at the tumor periphery (Figure 2).

\section{Discussion}

Distinction of primary melanoma and deep penetrating nevus was always the major topic when the deep penetrating nevus was investigated in the past. In the first study on deep penetrating nevi, Seab ${ }^{1}$ tried to make a distinction from melanoma using the proliferative capacity estimated by simply counting the number of mitoses. Lack of mitoses in deep penetrating nevi and the absence of a rapid clinical growth were suggested as critical features of the deep penetrating nevus. Also results from Mehregan $^{12}$ demonstrated subtle differences of PCNA expression in deep penetrating nevi seemingly corroborating Seab's data.

Consistent with our previous findings on the progressive increase of the major cell cycle switch pRb during melanoma development, ${ }^{13}$ semiquantitative immunoreactivity of $\mathrm{pRb}$ significantly outweighed in nodular malignant melanomas compared to deep penetrating nevi. However, in contrast to these former observations also reporting a progressive increase of phosphorylated, inactive $\mathrm{pRb}$, here, the differential expression of phosphopRb Ser795 lacked statistical significance, particularly due to broad intertumoral staining variations. Strikingly, compared to the results on common benign nevi, ${ }^{13}$ both $\mathrm{pRb}$ and phospho-pRb Ser795 showed a high deep dermal immunolocalization in a considerable subset of the deep penetrating nevus specimens indicating inactive $\mathrm{pRb}$, that is a cell cycle permissive status, very similar to nodular malignant melanoma.

Over the last years, MIB-1/Ki-67 expression has been repeatedly discussed to be correlated with disease progression and prognosis in large cohorts of melanoma patients. ${ }^{23,24}$ However, for differentiation of single indistinct cases of deep penetrating nevus from nodular malignant melanoma, this marker might practically not be applicable due to its high intertumoral expression variability, even in thick melanomas. ${ }^{24}$

Next to cellular proliferation, remodeling of the tumor-surrounding stroma by matrix metalloproteinases is considered as a most essential step for tumor progression. ${ }^{25}$ In accordance with previous 


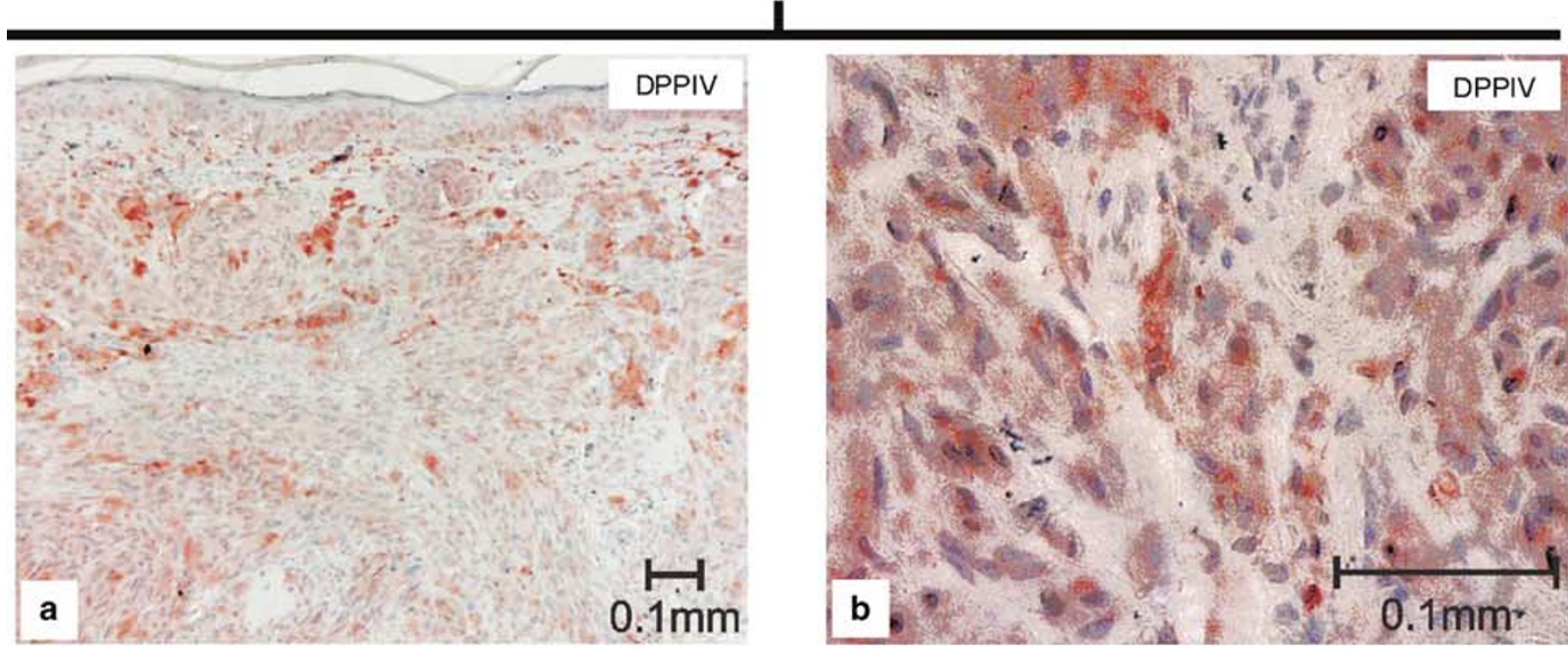

nodular malignant melanoma
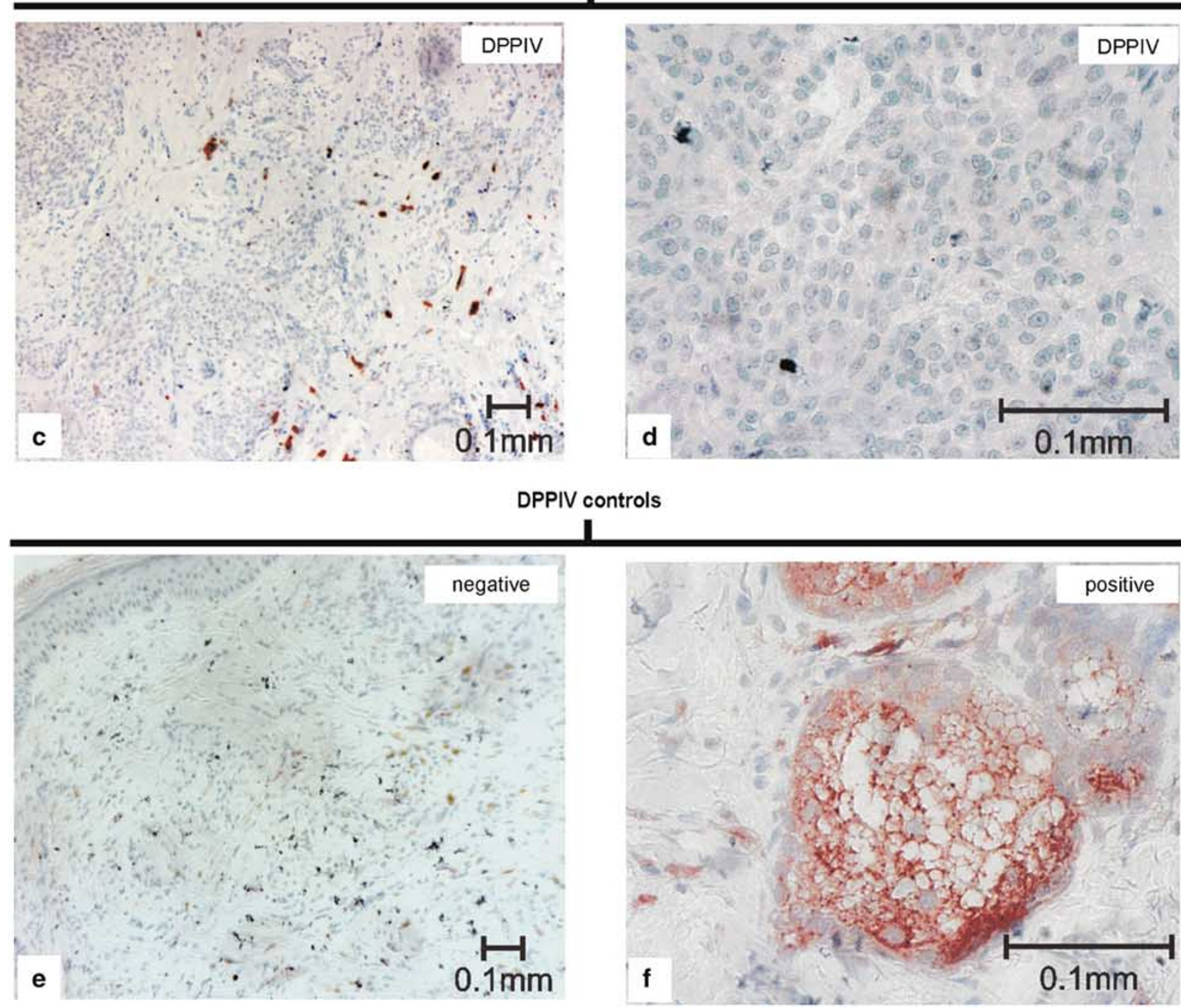

Figure 2 Immunoreactivity and immunolocalization of DPPIV in deep penetrating nevi vs nodular malignant melanomas. (a) DPPIV expression in a deep penetrating nevus specimen with its typical diffuse and, in part, focally accumulated staining pattern $(\times 100$ overview). (b) $\times 400$ magnification. (c) Example of a nodular malignant melanoma showing only single positive cells $(\times 100)$, $(\mathbf{d}) \times 400$ magnification. (e) Negative control ( $\times 100$ overview), (f) Positive control (sebaceous gland, $\times 400$ magnification). 
studies on MMP expression in melanomas, ${ }^{16,17,26}$ MMP-1, MMP-2, integrin $\beta 3$ and MT1-MMP were specifically overexpressed at the dermo-invasive front of nodular malignant melanomas also in this series. But, a more important result of this study is that most of these 'major suspects' considered as integral parts of melanoma progression leading to metastatic disease were not much different in deep penetrating nevi. After statistical evaluation of immunoreactivity, only MT1-MMP showed a trend to upregulation in nodular malignant melanomas. However, similar to most other invasion markers analyzed, it failed to show a differential immunoreactivity with statistical significance. Especially in the case of MT1-MMP, the lack of significance and, therefore, the inapplicability as a consistent discriminating marker is probably due to the broad variance of staining signals among the different tumor samples of both entities.

Most interestingly, only DPPIV kept its promise to be a protein that possibly discriminates true melanocytic malignancy and deep penetrating nevi. DPPIV is a cell surface peptidase expressed by normal melanocytes and common nevi, but primary and advanced malignant melanomas almost invariably lose or alter their DPPIV expression. ${ }^{20-22}$ These previous observations made DPPIV a prime candidate for this study. Functionally, DPPIV re-expression leads to a loss of tumorigenicity, anchorage-independent growth, redifferentiation and an acquired dependence on exogenous growth factors. ${ }^{22}$ Thus, high DPPIV expression was found correlated with less metastatic potential in vivo. ${ }^{27}$ The effect of DPPIV appears to be either mediated through the upregulation of other factors such as E-cadherin and tissue inhibitors of matrix metalloproteinases ${ }^{28}$ or by its ability to bind components of the extracellular matrix such as collagen or fibronectin. ${ }^{27,29,30}$

Beyond those practical implications of this study, our observations add to the evidence that the deep penetrating nevus could serve as a valuable, natural model of melanocytic progression and to dissect invasion mechanisms from metastatic potential. In this regard, the expression of MMP-1, MMP-2 was exceptional, since their immunoreactivity in deep penetrating nevi was unexpectedly high. In case of MMP-1, this upregulation in deep penetrating nevi even reached statistical significance. This is surprising since, the induction of MMP-1 has been suggested to be a pivotal late event in the progression of advanced melanomas not compatible with any benign condition ${ }^{16,26,31}$ and even dysplastic nevi as well as early melanomas usually lack a significant MMP-1 and MMP-2 expression. ${ }^{17,32,33}$

We conclude that among the 'major suspects' reflecting melanocytic tumor progress, DPPIV bears the highest potential to be further exploited as a marker in doubtful cases. Moreover, due to its emerging functional role in invasion/proliferation of various cancers, the molecular mechanisms downstream of DPPIV deserve to be studied in more detail.

\section{References}

1 Seab Jr JA, Graham JH, Helwig EB. Deep penetrating nevus. Am J Surg Pathol 1989;13:39-44.

2 Barnhill RL, Mihm Jr MC, Magro CM. Plexiform spindle cell naevus: a distinctive variant of plexiform melanocytic naevus. Histopathology 1991;18: 243-247.

3 Barnhill RL. Pathology and prognostic factors. Curr Opin Oncol 1993;5:364-376.

4 Robson A, Morley-Quante M, Hempel H, et al. Deep penetrating naevus: clinicopathological study of 31 cases with further delineation of histological features allowing distinction from other pigmented benign melanocytic lesions and melanoma. Histopathology 2003;43:529-537.

5 Mehregan DA, Mehregan AH. Deep penetrating nevus. Arch Dermatol 1993;129:328-331.

6 Ruiter DJ, van Dijk MC, Ferrier CM. Current diagnostic problems in melanoma pathology. Semin Cutan Med Surg 2003;22:33-41.

7 Barnhill RL, Barnhill MA, Berwick M, et al. The histologic spectrum of pigmented spindle cell nevus: a review of 120 cases with emphasis on atypical variants. Hum Pathol 1991;22:52-58.

8 Hoss DM, Grant-Kels JM. Significant melanocytic lesions in infancy, childhood, and adolescence. Dermatol Clin 1986;4:29-44.

9 Cooper PH. Deep penetrating (plexiform spindle cell) nevus. A frequent participant in combined nevus. J Cutan Pathol 1992;19:172-180.

10 Pulitzer DR, Martin PC, Cohen AP, et al. Histologic classification of the combined nevus. Analysis of the variable expression of melanocytic nevi. Am J Surg Pathol 1991;15:1111-1122.

11 Skelton III HG, Smith KJ, Barrett TL, et al. HMB-45 staining in benign and malignant melanocytic lesions. A reflection of cellular activation. Am J Dermatopathol 1991;13:543-550.

12 Mehregan DR, Mehregan DA, Mehregan AH. Proliferating cell nuclear antigen staining in deep-penetrating nevi. J Am Acad Dermatol 1995;33:685-687.

13 Roesch A, Becker B, Meyer S, et al. Overexpression and hyperphosphorylation of retinoblastoma protein in the progression of malignant melanoma. Mod Pathol 2005;18:565-572.

14 Roesch A, Becker B, Meyer S, et al. Retinoblastomabinding protein 2-homolog 1: a retinoblastoma-binding protein downregulated in malignant melanomas. Mod Pathol 2005;18:1249-1257.

15 Hofmann UB, Westphal JR, Waas ET, et al. Coexpression of integrin alpha(v)beta3 and matrix metalloproteinase-2 (MMP-2) coincides with MMP-2 activation: correlation with melanoma progression. J Invest Dermatol 2000;115:625-632.

16 Hofmann UB, Westphal JR, Van Muijen GN, et al. Matrix metalloproteinases in human melanoma. J Invest Dermatol 2000;115:337-344.

17 Hofmann UB, Westphal JR, Zendman AJ, et al. Expression and activation of matrix metalloproteinase-2 (MMP-2) and its co-localization with membrane-type 1 matrix metalloproteinase (MT1-MMP) correlate with melanoma progression. J Pathol 2000; 191:245-256.

18 Friedl P, Wolf K. Tumour-cell invasion and migration: diversity and escape mechanisms. Nat Rev Cancer 2003;3:362-374. 
19 Durko M, Navab R, Shibata HR, et al. Suppression of basement membrane type IV collagen degradation and cell invasion in human melanoma cells expressing an antisense RNA for MMP-1. Biochim Biophys Acta 1997;1356:271-280.

20 Houghton AN, Albino AP, Cordon-Cardo C, et al. Cell surface antigens of human melanocytes and melanoma. Expression of adenosine deaminase binding protein is extinguished with melanocyte transformation. J Exp Med 1988;167:197-212.

21 Morrison ME, Vijayasaradhi S, Engelstein D, et al. A marker for neoplastic progression of human melanocytes is a cell surface ectopeptidase. J Exp Med 1993; 177:1135-1143.

22 Wesley UV, Albino AP, Tiwari S, et al. A role for dipeptidyl peptidase IV in suppressing the malignant phenotype of melanocytic cells. J Exp Med 1999;190: 311-322.

23 Gimotty PA, Van Belle P, Elder DE, et al. Biologic and prognostic significance of dermal Ki67 expression, mitoses, and tumorigenicity in thin invasive cutaneous melanoma. J Clin Oncol 2005;23:8048-8056.

24 Hazan C, Melzer K, Panageas KS, et al. Evaluation of the proliferation marker MIB-1 in the prognosis of cutaneous malignant melanoma. Cancer 2002;95: 634-640.

25 MacDougall JR, Matrisian LM. Contributions of tumor and stromal matrix metalloproteinases to tumor progression, invasion and metastasis. Cancer Metastasis Rev 1995;14:351-362.

26 Airola K, Karonen T, Vaalamo M, et al. Expression of collagenases- 1 and -3 and their inhibitors TIMP-1 and -3 correlates with the level of invasion in malignant melanomas. Br J Cancer 1999;80:733-743.

27 Pro B, Dang NH. CD26/dipeptidyl peptidase IV and its role in cancer. Histol Histopathol 2004;19:13451351.

28 Kajiyama H, Kikkawa F, Khin E, et al. Dipeptidyl peptidase IV overexpression induces up-regulation of E-cadherin and tissue inhibitors of matrix metalloproteinases, resulting in decreased invasive potential in ovarian carcinoma cells. Cancer Res 2003;63: 2278-2283.

29 Abdel-Ghany M, Cheng H, Levine RA, et al. Truncated dipeptidyl peptidase IV is a potent anti-adhesion and anti-metastasis peptide for rat breast cancer cells. Invasion Metastasis 1998;18:35-43.

30 Pethiyagoda CL, Welch DR, Fleming TP. Dipeptidyl peptidase IV (DPPIV) inhibits cellular invasion of melanoma cells. Clin Exp Metastasis 2000;18:391400.

31 Yang Y, Dang D, Atakilit A, et al. Specific alpha v integrin receptors modulate K1735 murine melanoma cell behavior. Biochem Biophys Res Commun 2003;308:814-819.

32 Vaisanen A, Tuominen H, Kallioinen M, et al. Matrix metalloproteinase-2 (72 kD type IV collagenase) expression occurs in the early stage of human melanocytic tumour progression and may have prognostic value. J Pathol 1996;180:283-289.

33 Vaisanen A, Kallioinen M, Taskinen PJ, et al. Prognostic value of MMP-2 immunoreactive protein $(72 \mathrm{kD}$ type IV collagenase) in primary skin melanoma. J Pathol 1998;186:51-58. 